\title{
Landscape Analysis of Phase 2 and 3 Clinical Trials for Targeted Radionuclide Therapy
}

\author{
Erik Mittra ${ }^{1}$ and Lisa Bodei ${ }^{2}$ \\ ${ }^{I}$ Division of Nuclear Medicine and Molecular Imaging, Oregon Health \& Science University, Portland, Oregon; and \\ ${ }^{2}$ Molecular Imaging and Therapy Service, Memorial Sloan Kettering Cancer Center, New York, New York
}

W ithin nuclear medicine, theranostics has revitalized the field of targeted radionuclide therapy, and there are a growing number of investigator-initiated and industry-sponsored clinical trials of targeted radionuclide therapy. This article summarizes the current trials available in the National Institutes of Health database, the largest trial repository, to provide both an overview of the current landscape and a glimpse toward an undeniably exciting future for theranostics.

This landscape analysis was completed by searching for the terms "radionuclide therapy," "radioisotope therapy," "radiopharmaceutical therapy," and "radioligand therapy" on ClinicalTrials.gov in November 2020. Other terms may provide different results. Phase $1 / 2$, 2, and 3 trials that are currently recruiting and those not yet recruiting were included.

\section{STUDIES}

Overall, the results showed 42 clinical trials, including 13 in phase $1 / 2,26$ in phase 2 , and 3 in phase 3 . Given this range of phases, the planned enrollment varies widely from 10 to 813, with an average of 147 participants. Five different radioisotopes, 12 ligands or targets, and 11 different cancer types are represented (Fig. 1). These include 22 single- and 20 multiple-institution studies. Of the 42 total trials, 21 are single-group, 15 are randomized, and 6 are nonrandomized.

\section{MOLECULES AND TARGETS}

Of the 5 different radioisotopes represented, most (28 radioisotopes) use ${ }^{177} \mathrm{Lu}$. The next most common are ${ }^{131} \mathrm{I}$ (7 radioisotopes) and ${ }^{223} \mathrm{Ra}$ (6 radioisotopes). Others include 3 trials with ${ }^{90} \mathrm{Y}$ and 1 trial with ${ }^{67} \mathrm{Cu}$. The ligands or targets are also skewed, with 20 trials on somatostatin receptors. Of these, 16 are focused on DOTATATE/ oxodotreotide, 4 on DOTATOC/edotreotide, and 1 on MeCOSar$\mathrm{Tyr}^{3}$-octreotate (SARTATE; Clarity Pharmaceuticals). Expectedly, the next most common are the prostate-specific membrane antigen (PSMA) ligands (617, I\&T, R2, and MIP-1095) (1), with 8 trials, followed by 6 with ${ }^{223} \mathrm{Ra}$ for bone metastases and 4 with ${ }^{131}$ I-metaiodobenzylguanidine. The diversity of trials include ligands for human epidermal growth factor receptor 2, gastrin-releasing peptide receptor (bombesin), neurotensin receptor-1, B-cell CD37, and

\footnotetext{
Received Jan. 4, 2021; revision accepted Jan. 19, 2021

For correspondence and reprints contact: Erik Mittra, Oregon Health and Science University, 3181 S.W. Sam Jackson Park Rd., L340, Portland, OR 97239.

E-mail: mittra@ohsu.edu

Published online February 12, 2021. COPYRIGHT (C) 2021 by the Society of Nuclear Medicine and Molecular Imaging.
}

phospholipid ethers with the compound ${ }^{131}$ I-CLR1404 (2). The 11 cancer types of interest include gastroenteropancreatic neuroendocrine tumors (15 trials), prostate cancer (11 trials), and 4 trials each for pheochromocytoma or paraganglioma, neuroblastoma, or a variety of solid tumors. Less common are meningioma (3 trials), lymphoma ( 2 trials), and 1 trial each for Merkel cell carcinoma, breast cancer, renal cell cancer, and salivary gland carcinomas.

\section{DEVELOPMENTAL PHASE}

For phase 3 , there are only 3 trials. The largest is a multicenter (United States and Canada) trial sponsored by the Children's Oncology Group, with an enrollment of up to 813 participants, comparing ${ }^{131}$ I-iobenguane versus crizotinib plus standard therapy for younger patients with newly diagnosed high-risk neuroblastoma or ganglioneuroblastoma. The second is a single-site randomized trial (the ESCALATE trial at the Carolina Urologic Research Center) of 499 participants comparing ${ }^{223} \mathrm{Ra}$ plus enzalutamide or darolutamide versus enzalutamide or darolutamide alone for metastatic castration-resistant prostate cancer. The last is the worldwide (United States, Europe, South Africa, and Australia) COMPETE trial, sponsored by Isotopen Technologien Munchen AG. This is a multisite, randomized trial of 300 participants with gastroenteropancreatic neuroendocrine tumors. The primary goal is comparison of ${ }^{177} \mathrm{Lu}$-edotreotide to everolimus.

Phase 2 includes the largest number of trials (26 trials) and the largest spectrum of design. These trials include novel agents for expected indications, novel or off-label indications for approved agents, and questions of combining or sequencing approved therapies. Examples of novel agents include ${ }^{177} \mathrm{Lu}$-PSMA-I\&T and ${ }^{131}$ I-MIP-1095 for prostate cancer or ${ }^{131}$ I-CLR1404 for lymphoma. Examples of novel or off-label indications include ${ }^{177}$ Lu-PSMA for salivary gland carcinomas and peptide receptor radionuclide therapy (PRRT) for non-gastroenteropancreatic neuroendocrine tumors (e.g., pheochromocytoma or paraganglioma and thymic neuroendocrine tumors) or meningioma. Examples of combining or sequencing existing therapies include neoadjuvant PRRT for pancreatic neuroendocrine tumors, ${ }^{223} \mathrm{Ra}$ plus paclitaxel for breast cancer or in addition to cabozantinib for renal cell cancer, capecitabine or temozolomide plus PRRT for gastroenteropancreatic neuroendocrine tumors, and PRRT versus sunitinib for pancreatic neuroendocrine tumors. There are also several studies on dosimetry-based personalization of PRRT.

Phase 1/2 has 13 studies, with a focus on novel radiopharmaceuticals. These include ${ }^{177} \mathrm{Lu}$-lilotimab satetraxetan targeting CD37 for 


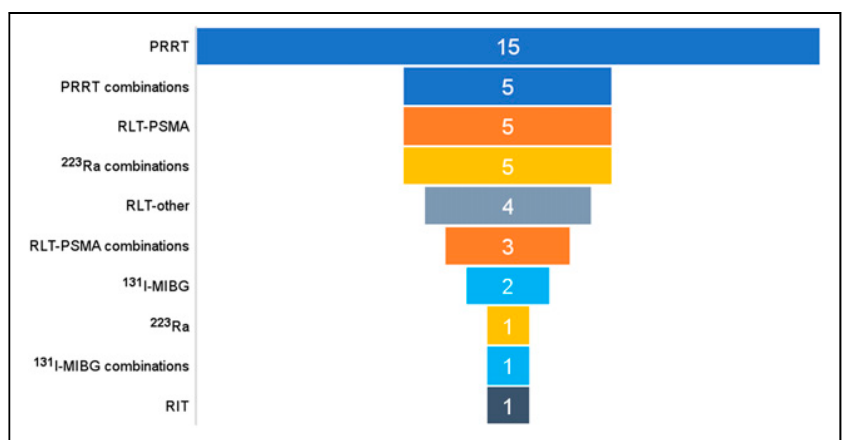

FIGURE 1. Clinical trials for targeted radionuclide therapy, most of which involve PRRT (15 studies) or PRRT combinations (5 studies) predominantly for neuroendocrine neoplasms, mainly with ${ }^{177}$ Lu-DOTATATE (16 studies). Radioligand therapy with PSMA agents, alone ( 5 studies) or in combination (3 studies), and ${ }^{223}$ Ra-dichloride, mainly in combinations (5 studies), account for most remaining studies, directed mainly at prostate cancer.

${ }^{131} \mathrm{I}$-MIBG $={ }^{131} \mathrm{I}$-metaiodobenzylguanidine; other = radiolabeled bombesin, neurotensin, phospholipid ester and human epidermal growth factor receptor 2 ligands, and anti-CD37 antibodies; ${ }^{223} \mathrm{Ra}={ }^{223} \mathrm{Ra}$-dichloride; $\mathrm{RIT}=$ radioimmunotherapy; $\mathrm{RLT}=$ radioligand therapy.

follicular lymphoma; ${ }^{131} \mathrm{I}-\mathrm{CAM}-\mathrm{H} 2$ (3) for breast, gastric, and gastroesophageal junction cancers; ${ }^{67} \mathrm{Cu}$-SARTATE for neuroblastoma; ${ }^{177} \mathrm{Lu}$-PSMA-R2 for metastatic castration-resistant prostate cancer; and ${ }^{177} \mathrm{Lu}$-neobombesin and ${ }^{177} \mathrm{Lu}-3 \mathrm{BP}-227$ (a neurotensin receptor 1 agonist) for a variety of solid cancers. Similar to phase 2 , other trials investigate novel indications for approved agents and questions of combining or sequencing approved therapies such as the combination of ${ }^{177} \mathrm{Lu}$-DOTATATE and ${ }^{131}$ I-metaiodobenzylguanidine for midgut neuroendocrine tumors, the role of ${ }^{177} \mathrm{Lu}-\mathrm{PSMA}-617$ before prostatectomy, the combination of ${ }^{177} \mathrm{Lu}-$ PSMA and immunotherapy for prostate cancer, and the combination of olaparib and ${ }^{223}$ Ra-dichloride for prostate cancer. There are also additional studies on dosimetry-guided PRRT.

\section{CONCLUSION}

This landscape analysis, although not exhaustive and only a snapshot in time, provides an overview of phase $1 / 2,2$, and 3 studies of targeted radioisotope therapies. As seen, PRRT, radioligand therapy, and their variations are predominant. Additionally, there are multiple new targets or ligands - and, as a result, cancer types - being evaluated with a variety of agents using the theranostics approach. Furthermore, for agents that are already approved or are soon to be approved, considerable work is being done to expand the indications and improve their efficacy using combination therapies, better understanding of sequencing of therapies, and enhancement of the personalized approach using dosimetry. Most multicenter trials and some single-center trials are sponsored by pharmaceutical companies, a critical factor in bringing these trials to fruition and advancing the field. This analysis highlights the concentration of studies and ideas around the concept of theranostics while these trials continue to grow and the field further matures.

\section{DISCLOSURE}

No potential conflict of interest relevant to this article was reported.

\section{ACKNOWLEDGMENT}

We thank Amanda Abbott, of the Clinical Trials Network at the Society of Nuclear Medicine and Molecular Imaging, Reston, Virginia, for assistance with this analysis.

\section{REFERENCES}

1. Lütje S, Slavik R, Fendler W, Herrmann K, Eiber M. PSMA ligands in prostate cancer: probe optimization and theranostic applications. Methods. 2017;130:42-50.

2. Marsh IR, Grudzinski J, Baiu DC, et al. Preclinical pharmacokinetics and dosimetry studies of ${ }^{124} \mathrm{I} /{ }^{131} \mathrm{I}$-CLR1404 for treatment of pediatric solid tumors in murine xenograft models. J Nucl Med. 2019;60:1414-1420.

3. Matthias D'Huyvetter M, De Vos J, Caveliers V, et al. Phase I trial of ${ }^{131}$ I-GMIB-AntiHER2-VHH1, a new promising candidate for HER2-targeted radionuclide therapy in breast cancer patients. $J$ Nucl Med. December 4, 2020 [Epub ahead of print]. 